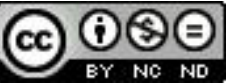

\section{Educação Estatística no Curso de Licenciatura em Matemática}

\section{Statistics Education in Undergraduate Mathematics}

\author{
Celi Espasandin Lopes*
}

\begin{abstract}
Resumo
Este artigo apresenta uma discussão sobre a disciplina de Estatística no curso de formação inicial do futuro professor de matemática que atuará na Educação Básica. Tem-se por objetivo problematizar o desenvolvimento teórico e metodológico dessa disciplina na formação docente, a partir da experiência de estágio na disciplina de Estatística oferecida no segundo semestre de 2008, na Universidade da Georgia (The University of Georgia). A proposta da disciplina parte da concepção de que estatística é uma ciência de análise de dados e que não pode ser tratada em um curso de formação de professores como uma parte da matemática aplicada. O desenvolvimento metodológico da disciplina centra-se na resolução de problemas e no uso de recursos tecnológicos. Os resultados evidenciam que as atividades de ensino propostas no curso promovem o desenvolvimento do pensamento estatístico dos futuros professores de matemática e auxilia-os na percepção sobre o trabalho a ser desenvolvido com seus futuros alunos. Cabe ressaltar, também, a importância da interação de futuros professores com professores em serviço durante as aulas regulares da disciplina.
\end{abstract}

Palavras-chave: Ensino Superior. Matemática. Estatística. Educação.

\begin{abstract}
This article presents a discussion on Statistics courses in undergraduate education of future math teachers who will work at the elementary school level. It aims to discuss the

\footnotetext{
* Doutora em Educação, Universidade Estadual de Campinas (UNICAMP). Docente do Programa de Pós-Graduação em Educação da Universidade São Francisco (USF), Itatiba, São Paulo, Brasil. Docente do Doutorado em Ensino de Ciências e Matemática da Universidade Cruzeiro do Sul (UNICSUL), São Paulo, São Paulo, Brasil. Endereço para correpondência: Av. Gessy Lever, 915/383, Valinhos, CEP: 13272-000, São Paulo, Brasil.E-mail: celilopes@uol.com.br.
} 
theoretical and methodological development of this course in teacher education based on the internship experience in statistics courses offered in the second semester of 2008 at the University of Georgia. The proposal of the course is based on the conception that statistics is a science of data analysis and cannot be addressed in a teacher education program as part of applied mathematics. The methodological development of the course focuses on problem solving and the use of technological resources. The results show that the proposed learning activities in the course promote the development of statistical thinking of future mathematics teachers and helps them in their perception regarding the work to be carried out with future students. It is also worth noting the importance of the interaction of future teachers with in-service teachers during the classes.

Keywords: Undergraduate. Mathematics. Statistics. Education.

\section{Introdução}

Este novo século que estamos vivendo foi definido como a Era da Informação, pois os dados estão em toda parte. A análise de dados se tornou uma componente-chave no currículo, desde a educação infantil até o ensino superior. Estamos rodeados de dados coletados a partir de levantamentos e experimentos, as duas formas de pesquisa empírica. A pesquisa empírica é qualquer investigação em que os dados são recolhidos a partir do mundo externo e, em seguida, conclusões são tiradas a partir deles.

Essa realidade remete a repensares sobre o currículo da escola e da universidade. Os currículos de matemática têm apontado para a necessidade de iniciar uma educação estatística já nos primeiros anos de escolaridade, e isso requer que os futuros professores tenham uma formação adequada para realizar tal trabalho.

Atualmente, o professor trabalha com um grupo diferente de alunos, que têm acesso a estatísticas mais cedo em suas vidas e necessitam tomar decisões rapidamente. Aquela formação matemática, que se centrava em fórmulas, algoritmos e exercícios de fixação, tornou-se insuficiente.

Lopes (2008) já afirmava que a formação dos professores não incorporava um trabalho sistemático sobre estocástica, dificultando a possibilidade de esses profissionais desenvolverem um trabalho significativo com essa temática nas salas de aula da educação básica. A autora apontava a necessidade de a formação do educador matemático prever um processo de ensino e aprendizagem de conteúdos pela resolução de problemas, por simulações e experimentos, de forma a permitir ao profissional construir conhecimentos, à medida que 
estabelece relações com informações adquiridas e com o domínio de diferentes linguagens e formas de expressão.

Ainda hoje, essas questões estão em pauta nas produções científicas de educação matemática e educação estatística, o que justifica as discussões que se apresentam neste texto. Vale analisar alguns aspectos emergentes nas pesquisas sobre a educação estatística na formação dos professores no Brasil, para, depois, discutir a experiência de uma universidade americana.

\section{A estatística na licenciatura de matemática}

No cenário nacional, a produção científica em educação estatística ampliou-se muito na primeira década do século XXI, haja vista que o periódico Bolema, classificado na lista Qualis como A1, publicou, em 2011, duas edições temáticas de educação estatística. No entanto, as pesquisas sobre a disciplina de estatística nos cursos de licenciatura em matemática ainda são poucas, mas já revelam aspectos importantes a serem refletidos.

$\mathrm{Na}$ pesquisa realizada por Bayer et al. (2004), perguntou-se a 80 formandos em matemática, de nove estabelecimentos de ensino superior do Rio Grande do Sul, se o entrevistado considerava ter tido um curso de matemática que o tivesse preparado bem para lecionar estatística. Embora aproximadamente $71 \%$ tivessem expressado que consideravam importantes os conteúdos estatísticos, $52 \%$ afirmaram que não tiveram formação adequada para lecionar estatística. Outro dado interessante dessa pesquisa é que 46,3\% dos futuros professores de matemática entrevistados indicaram como maior dificuldade para lecionar estatística a falta de conhecimentos suficientes e, aproximadamente $39 \%$ apontaram a ausência de material didático como a maior dificuldade.

Esses dados, embora específicos de um determinado estado brasileiro, expressam muito da realidade nacional. Não apenas os alunos da licenciatura em matemática se sentem despreparados para abordar a estatística nas aulas de matemática da educação básica, mas a ausência de material didático que subsidie o trabalho docente é ampla. O mesmo ocorre com livros-textos de ensino superior, que, em sua maioria, são traduzidos de outros países, pois a produção nacional ainda não tem se dedicado a publicações específicas para o curso de licenciatura em matemática.

Esse problema, em suas duas faces, aparece evidenciado por Costa e Nacarato (2011), que afirmam que os conteúdos de estatística desenvolvidos nos cursos de licenciatura a partir das ementas - pensadas, muitas vezes, para 
todos os cursos de graduação - não contemplam o movimento do pensamento crítico e o desenvolvimento da pesquisa ou da investigação, tornando o aprendizado estanque e não transformador, ao contrário do que se desejava. E revelam, também, que grande parte dos livros-textos para os cursos de estatística no ensino superior assumem uma perspectiva de estatística aplicada, para atender aos cursos relacionados à area de finanças ou saúde. Vale destacar que as considerações das autoras decorrem da análise de uma pesquisa de mestrado da qual evidenciamos, a seguir, outros resultados - que apresentou significativa construção de dados sobre a estatística na formação do professor de matemática.

O estudo de Costa (2007) destaca-se nas pesquisas brasileiras sobre educação estocástica na formação do professor de matemática, por ter investigado a percepção dos professores em exercício na educação básica e dos formadores de professores no ensino superior. É importante ressaltar que as entrevistas foram realizadas com educadores estatísticos de significativa produção científica, que atuam na formação inicial de professores de matemática. Esses entrevistados revelam que reconhecem a existência de lacunas na formação docente, bem como problemas conceituais e epistemológicos nos livros didáticos; destacam, também, a pouca flexibilidade nas atuais ementas dos cursos de licenciatura; e sinalizam a importância de sua reformulação, de forma a atender às necessidades da formação do pensamento estatístico nos futuros professores, para que possam atuar com segurança na educação básica.

Corroborando essas ideias, há Costa e Pamplona (2011), que alertam para a importância de compreender que cabe ao curso de licenciatura em matemática a formação não só de educadores matemáticos, mas também de educadores estatísticos. Esses autores consideram que tal visão requer uma modificação no repertório do professor de matemática, e defendem que os cursos de formação de professores de matemática gerem espaços para discussões acerca de probabilidade e estatística, não só partilhando conhecimentos específicos, mas comunicando e suscitando reflexões sobre experiências com o ensino dessas temáticas na educação básica.

Diante desse contexto, cabe destacar a importância de ampliar a discussão sobre a disciplina de estatística para o curso de licenciatura em matemática, visando atrelar as considerações sobre a formação necessária para o futuro professor de matemática ao que têm revelado as pesquisas sobre educação estatística para a educação básica. 


\section{Educação estatística e a formação do professor de matemática}

Um primeiro entendimento necessário é de que a estatística é uma ciência distinta da matemática e, portanto, seus objetos de estudo são diferenciados. Concordamos com Cobb e Moore (1997), que partem da seguinte tese: a estatística tem forças culturais que podem ajudar muito na matemática, enquanto a matemática tem forças organizacionais que podem fornecer abrigo para a estatística acadêmica. Para eles, a estatística é uma disciplina metodológica que não existe para si, mas, sim, para oferecer aos outros campos de estudo um conjunto coerente de ideias e ferramentas para lidar com dados.

A estatística fornece meios para lidar com dados que levem em conta a onipresença da variabilidade, o que a diferencia, significativamente, da matemática e de outras ciências. Outro aspecto que distingue o pensamento estatístico do pensamento matemático é que a ciência estatística requer um tipo diferente de pensar, porque os dados são não apenas os números, eles são números com um contexto.

Os matemáticos, muitas vezes, dependem do contexto tanto para a motivação como para gerar problemas para a pesquisa. Para Cobb e Moore (1997), o foco principal no pensamento matemático centra-se em padrões abstratos, ou seja, o contexto é parte do detalhe irrelevante que deve ser fervura sobre a chama da abstração, a fim de revelar o oculto. Em matemática, o contexto obscurece a estrutura. Como os matemáticos, os analistas de dados também procuram por padrões, mas, em última instância, na análise de dados, isso ocorre se os padrões têm significado e estabelecem relações com a temática do problema investigado. $\mathrm{Na}$ análise dos dados, o contexto fornece o significado.

Essa diferença tem profundas implicações para o ensino. Para ensinar estatística, não é suficiente entender a teoria matemática e os procedimentos estatísticos; é preciso fornecer ilustrações reais aos estudantes e saber como usá-las para envolver os alunos no desenvolvimento de seu juízo crítico.

O ensino de estatística em um curso de licenciatura de matemática precisa, não apenas, ter o o quê, o porquê, o quem e o quando, mas, essencialmente, o como. Apesar de muito se ter discutido nos últimos anos sobre como os alunos devem aprender estatística, é necessário pensar como nós, os professores, podemos ser mais eficazes em promover a aprendizagem de nossos estudantes.

A American Statistical Association (ASA) financiou o projeto para a elaboração das Diretrizes para Avaliação e Ensino em Educação Estatística, o GAISE Project, que consiste em dois grupos: um, concentrado em 
recomendações para a educação básica, publicado em 2007, tendo os trabalhos sido coordenados por Christine Franklin (FRANKLIN et al., 2007); e outro, para o ensino superior, publicado em 2010 e elaborado segundo a coordenação de Joan Garfield (ALIAGA et al., 2010).

O documento Gaise Report College (ALIAGA et al., 2010) lembra que alguns professores oferecem cursos que são fortemente ministrados com o objetivo de os alunos se tornarem consumidores estatisticamente alfabetizados e com domínio sobre os dados. Afirma que outros cursos são ministrados para ensinar os estudantes a tornarem-se produtores de análises estatísticas.

Declara, igualmente, que os cursos, na maioria, são uma mistura de componentes de consumo e produção. A partir dessas observações, o documento recomenda que o resultado proposto para todos os cursos de introdução estatística seja produzir alunos educados estatisticamente, o que significa que os estudantes devem desenvolver a literacia estatística e a capacidade de pensar estatisticamente.

Para isso, o Gaise Report College sugere seis metas que devem ser consideradas no trabalho com os alunos: enfatizar literacia estatística e desenvolver o pensamento estatístico; usar dados reais; ressaltar o entendimento conceitual, ao invés de mero conhecimento de procedimentos; promover a aprendizagem ativa em sala de aula; usar a tecnologia para o desenvolvimento de compreensão conceitual e análise de dados; e usar as avaliações para melhorar e avaliar a aprendizagem do aluno.

$\mathrm{Na}$ primeira meta: enfatizar literacia estatística e desenvolver o pensamento estatístico, considera-se a literacia estatística como a compreensão da linguagem básica da estatística e de suas ideias fundamentais. E define-se o pensamento estatístico como o tipo de pensamento que os estatísticos usam, quando reconhecem a variação presente no processo, utilizam métodos e ferramentas estatísticas para quantificar e entender a variação, resolvem problemas estatísticos. Os autores do documento destacam que o pensamento estatístico tem sido caracterizado pela necessidade de dados, pela importância dos dados de produção, pela onipresença de variabilidade e pela quantificação e explicação da variabilidade.

Alertam, ainda, os autores, para a importância de usar dados reais nas aulas de estatística, para que a tarefa seja autêntica e considere as questões relacionadas a como e por que os dados foram produzidos ou recolhidos; e de relacionar a análise com o contexto do problema. Sugerem que se use um conjunto de dados reais, de interesse para os alunos, pois isso também é uma boa maneira 
de envolvê-los na reflexão sobre os dados relevantes e os conceitos estatísticos. Existem muitos tipos de dados reais: os disponibilizados em arquivos; os gerados na própria sala de aula; e os dados simulados. Um aspecto importante, ao lidar com dados reais, é ajudar os estudantes a formular boas perguntas e usar os dados para respondê-las de forma adequada, com base em como foram produzidos.

Em relação a ressaltar o entendimento conceitual, ao invés de mero conhecimento dos procedimentos, o documento destaca a preocupação com cursos que trazem muito material, o que leva os alunos a uma compreensão superficial dos conceitos. Se eles não compreenderem os conceitos importantes, haverá pouco valor em conhecer um conjunto de procedimentos. Se compreenderem bem os conceitos, então os procedimentos particulares serão fáceis de aprender. É preciso reconhecer que dar mais atenção aos conceitos do que aos procedimentos pode ser difícil politicamente, tanto para os professores quanto para os estudantes. No entanto, os alunos com uma boa base conceitual estarão bem preparados para estudar outras técnicas estatísticas, como métodos de pesquisa, regressão, desenho experimental ou métodos estatísticos em um segundo curso.

$\mathrm{Na}$ quarta recomendação - promover a aprendizagem ativa em sala de aula -, os autores sugerem que se utilizem metodologias ativas de aprendizagem em sala de aula e indicam como uma forma valiosa para promover a aprendizagem colaborativa o trabalho em grupos, que permita aos alunos aprender uns com os outros. Defendem que a aprendizagem ativa permite aos alunos descobrir, construir e compreender as ideias estatísticas e modelar o pensamento estatístico. Atividades desafiadoras têm um benefício adicional, na medida em que envolvem os alunos na aprendizagem e tornam divertido o processo de aprendizagem.

Cabe esclarecer que os princípios da aprendizagem ativa indicam que os alunos aprendem mais quando incentivados a discutir, a refletir e a resolver os problemas; e tiram proveito das ocasiões para pôr em prática suas ideias e os conhecimentos do conteúdo juntamente com os colegas. Os alunos adquirem facilmente novos conhecimentos, quando estão relacionados a experiências verídicas, a exemplos pessoais e a noções já aprendidas; e aprendem melhor e alargam os conhecimentos, quando efetuam tarefas autênticas, que se apoiam no que já sabem. Também, aprendem mais quando têm certo controle sobre o que aprendem e sobre a forma como aprendem (BRICKMAN; TAYLOR, 1996).

Já a recomendação feita pelo documento - usar a tecnologia para o 
desenvolvimento de conceitos e análise de dados - é uma das mais importantes, pois a tecnologia alterou a forma do trabalho estatístico, e, por isso, deve-se mudar o que ensinar em estatística e como fazê-lo. O documento considera que deve ser usada a tecnologia - ao invés de ferramentas computacionais mecânicas - para analisar dados, permitir aos alunos concentrarse na interpretação dos resultados e testes de condições. Do mesmo modo, os recursos tecnológicos devem ser empregados para ajudar os estudantes a visualizar conceitos e desenvolver a compreensão de ideias abstratas por simulações. Algumas ferramentas oferecem os dois tipos de usos, enquanto, em outros casos, um pacote de software estatístico pode ser completado por applets $w e b^{l}$. Independentemente das ferramentas utilizadas, um software é importante para visualizar o uso da tecnologia não apenas como uma maneira para calcular números, mas como uma forma de explorar ideias conceituais e melhorar a aprendizagem dos alunos.

Usar as avaliações para melhorar e avaliar a aprendizagem do aluno é uma recomendação importante, pois, na concepção do documento, os alunos irão valorizar o que o professor avaliar. Portanto, as avaliações precisam ser alinhadas com os objetivos de aprendizagem. Devem ser formativas e resultantes de vários instrumentos, para que o professor e o aluno tenham uma visão ampla sobre a aprendizagem estatística.

A partir das recomendações desse documento, vamos analisar o curso de estatística elaborado e ministrado pela professora Christine Franklin, na Universidade da Georgia.

\section{0 curso STAT 4/6070 e uma educadora estatística em ação}

A Universidade da Georgia (The University of Georgia - UGA), localizada em Athens, estado da Georgia, nos Estados Unidos, foi fundada em 1785. Atualmente, possui cerca de 33 mil alunos. É composta por 14 faculdades, dentre elas a Faculdade de Educação - a maior do país -, que conta com o Departamento de Educação Matemática. O Departamento de Estatística está alojado na Faculdade de Artes e Ciências, que é a maior da UGA.

Franklin revela que, na UGA, o trabalho interdisciplinar é fortemente encorajado e apoiado. Os Departamentos de Matemática e Educação Matemática têm trabalhado cooperativamente por muitos anos e proporcionado

\footnotetext{
${ }^{1}$ Applet é um software aplicativo que é executado no contexto de outro programa (como, por exemplo, um web browser); uma applet geralmente executa funções bem específicas.
} 
a formação de professores em matemática. Em 1996, os Departamentos de Estatística e Educação Matemática começaram a trabalhar em conjunto, especificamente voltados ao desenvolvimento de um novo curso de Estatística e Probabilidade para futuros professores de Matemática. Nasceu, então, o STAT 4/6070, curso ministrado duas vezes por ano e oferecido aos graduandos em Matemática e aos professores em exercício que fazem pós-graduação (FRANKLIN, 2002). Sua proposta associa conteúdo estatístico e conteúdo didático, a fim de preparar professores para o futuro trabalho docente de qualidade, nos cursos de Estatística a serem ministrados no ensino secundário.

No primeiro dia do curso, a professora entregou aos estudantes seu programa de trabalho com os alunos, com a citação: Statistical thinking will one day be as necessary for eficiente citizenship as the ability to read and write. H. G. Wells. Em Lopes (1998, p.31), esse pensamento está traduzido como "O pensamento estatístico um dia será tão necessário para o exercício eficiente da cidadania como a capacidade de ler e escrever". O visionário escritor britânico, Herbert George Wells (1866-1946), explicitou uma ideia, no início do século passado, que não poderia ser mais atual.

O STAT 4/6070 é um curso de estatística projetado para:

- ser um segundo curso de estatística, reforçando os conceitos e as técnicas aprendidas em um curso introdutório; apresenta discussões pedagógicas para o ensino de análise de dados e introduz temas estatísticos para além dos oferecidos no curso introdutório;

- enfatizar o pensamento estatístico e discutir como a estatística é usada em situações cotidianas;

- dar uma apreciação duradoura do papel vital do domínio da estatística na pesquisa empírica;

- compreender a importância de incluir a análise de dados no currículo de matemática. Especificamente, para professores do ensino secundário da Georgia, oferece preparação para ensinar o componente de análise de dados nos padrões de desempenho definidos no currículo de matemática da Georgia;

- ser ministrado com entrelace do conteúdo estatístico com a abordagem pedagógica.

O programa do curso determinou como elementos básicos do pensamento estatístico:

- a necessidade de aprender a lidar com dados. É importante reconhecer a necessidade de embasar as decisões e as ações pessoais 
em evidências (dados) e de reconhecer o perigo potencial de atuar em suposições não apoiadas por dados;

- a importância da produção de dados e obtenção de dados precisos. A causa mais frequente de uma amostra pobre é a falta de projeto antes de coletar os dados. Investigações bem planejadas para responder a perguntas específicas são a parte mais importante do fazer estatístico, porque, se não for bem feita, toda a análise dos dados resultantes não vai valer muito;

- o papel da variabilidade. Variabilidade é a essência de estatísticas!;

- a quantificação e a explicação da variabilidade. Isso envolve a compreensão do papel da aleatoriedade, usando distribuições para resumir dados, procurando neles padrões e desvios e utilizando modelos matemáticos para descrever os padrões e as relações.

Para que os alunos pudessem se apropriar desses elementos, o curso foi organizado com onze tópicos. O primeiro visava ao uso da estatística para $a$ tomada de decisões com relação a informações. $\mathrm{O}$ segundo era a exploração de dados com gráficos e resumos numéricos, seguido do tópico de coleta de dados. O quarto tópico se referia ao uso da probabilidade para descrever o comportamento aleatório e analisar como isso afeta nossas vidas diárias. $\mathrm{O}$ quinto tópico abordava as distribuições de probabilidade e as distribuições de amostragem, seguido de inferência estatística: estimação. O sétimo tópico era o poder de um teste; o oitavo tratava de inferência baseada em duas amostras; em seguida, inferências sobre a relação entre duas variáveis; e, finalmente, regressão.

A professora, autora da proposta, considerava que o curso tinha que discutir a estatística na prática, e que isso envolve a compreensão sobre as características de um problema e o saber selecionar e aplicar a ferramenta estatística mais adequada para responder ao problema. No ensino de estatística é mais importante se concentrar no entendimento a partir de dados, usando um julgamento e resolução de problemas, ao invés de simplesmente aplicar fórmulas para calcular números, pois a prioridade está sobre o desenvolvimento do pensamento estatístico. O uso de fórmulas deve ser secundário, sua escolha deve ser feita por uma razão específica e ela deve levar a uma conclusão adequada.

Esses pressupostos marcaram o curso desenvolvido no outono de 2008. A professora fez uso da estatística como apresentado na literatura atual, em casos de artigos de jornais e revistas. Ela desafiava os alunos, em todas as 
aulas, a pensar estatísticamente sobre assuntos diversos, como campeonato de futebol, campanhas eleitorais, dados econômicos e todo tipo de assunto em destaque na mídia. O uso da tecnologia era constante nas aulas, pelo uso da calculadora (TI-83+/84) ou do software Minitab, recursos que permitiam a análise de dados exploratória, simulações e a execução das técnicas de inferência estatística, bem como a simulação de alguns conceitos de inferência estatística.

Em várias aulas, as atividades eram realizadas em grupos. A professora apresentava problemas estatísticos que deveriam ser resolvidos pelo debate entre os participantes. Esse também era um momento ímpar do curso, pois os grupos eram formados por futuros professores que faziam a licenciatura em matemática e por professores em exercício que cursavam a pós-graduação. Os enunciados dos problemas eram muito bem elaborados e alguns eram do livro-texto adotado - Statistics: The art and science of learning from data -, de autoria de Alan Agresti e Christine Franklin. Cabe ressaltar que, sendo a professora coautora, sua proposta de trabalho está muito integrada à do livro-texto, que tem como fio condutor a resolução de problemas em estatística.

Dentre as propostas do curso se destacavam, ainda, atividades e projetos com o objetivo de reforçar e desenvolver conceitos estatísticos.

O curso teve como principais objetivos a aprendizagem de: como ler dados criticamente e com compreensão; como produzir dados que forneçam respostas claras para as perguntas corretamente colocadas; métodos sólidos e adequados para a elaboração de conclusão confiável e baseada em dados; como fazer descrições defensáveis e inferências sobre amostras e populações; como comunicar as conclusões de estudos estatísticos; e, como usar os recursos disponíveis para produzir atividades de ensino em estatística e probabilidade para educação básica.

A professora explicitou suas expectativas aos alunos no primeiro dia de aula. Disse a eles que gostaria que eles assistissem às aulas acordados e participando ativamente; que lessem o material solicitado para cada aula; que realizassem as propostas no tempo solicitado; que tivessem cortesia em sala de aula; e que não se atrassassem para o início da aula, nem se ausentassem antes do término e, caso necessitassem fazê-lo, deveriam justificar à professora. Avisou que não seria tolerada trapaça de qualquer natureza e que esse não seria considerado um delito light. Esperava que os alunos se esforçassem ao máximo e apreciassem o estudo da estatística. Explicitou, igualmente, suas próprias expectativas em relação a si mesma: preparar e desenvolver o melhor possível seu curso, criar um ambiente confortável de aprendizagem na sala de aula, estar 
disponível para ajudar e responder a questões constantemente e providenciar gabaritos para auxiliar os alunos em trabalhos práticos.

Como processo avaliativo, determinou que a nota máxima seria distribuida da seguinte forma: testes (20\%); prova $1(20 \%)$; prova $2(25 \%)$; atividades (livro-texto, tecnologias, projetos) 20\%; portfólio (15\%).

A elaboração do portfólio foi muito interessante, pois era o principal momento para os alunos apropriarem-se de discussões didáticas sobre o ensino de estatística e probabilidade na educação básica, já que deveriam buscar atividades e artigos relacionados à ação docente.

O desenvolvimento do curso levou os futuros professores não só a acreditar e entender o porquê a variabilidade é natural, previsível e quantificável, mas, também, a perceber que a amostragem aleatória permite que resultados de pesquisas e experimentos possam ser extrapolados para a população da qual a amostra foi tomada. Essa aprendizagem estatística revela um domínio sobre o conhecimento estatístico essencial a um profissional que terá o desafio de promover o desenvolvimento do pensamento estatístico de inúmeros jovens.

\section{Considerações}

As discussões apresentadas no texto, a partir de pesquisas realizadas, de documentos analisados e da observação do desenvolvimento de um curso de estatística, encaminham a considerar a urgência de repensar a programação e o desenvolvimento dessa disciplina nos cursos de licenciatura em matemática, se quisermos que a educação estocástica se efetive na formação de nossos estudantes da educação básica.

Os futuros professores precisam obter uma formação estatística que lhes permita pensar estatisticamente e aprender como promover o desenvolvimento do pensamento estatístico de seus futuros alunos. Para isso, a programação do curso de estatística para a licenciatura precisa ser revista e deve possibilitar aos alunos a apropriação de um conhecimento estatístico que vá além da resolução de problemas, ou seja, deve promover a realização de projetos e atividades de investigação e a problematização de situações diversas; e escolher adequadamente os processos de coleta, representação e análise de dados.

Podem-se considerar relevantes as etapas sugeridas no documento Gaise Report College para pensar as propostas de disciplinas de Estatística nos cursos de Licenciatura em Matemática, pois a análise do curso, exposta neste artigo, 
revelou que a articulação do uso de tecnologias à resolução de problemas estatísticos possibilitou aos futuros professores de Matemática uma significativa aquisição de conceitos e procedimentos. No trabalho pedagógico desenvolvido, houve ênfase nos processos de literacia estatística, propostas que promoveram o desenvolvimento do pensamento estatístico por meio do uso constante de dados reais. A elaboração de um portifólio com o objetivo de atrelar a aprendizagem estatística à aprendizagem docente permitiu a sistematização do entendimento conceitual; as tarefas desenvolvidas em grupo, a partir de situações problema, oportunizaram discussões sobre a diversidade de procedimentos possíveis para a solução de um mesmo problema, gerando uma aprendizagem ativa em sala de aula. O uso constante de calculadora gráfica e de software estatístico possibilitou a compreensão conceitual e a análise de dados, e o processo avaliativo, com diversidade de instrumentos utilizados, a cada aula do curso, permitu ao aluno mapear suas dificuldades e potencialidades e recorrer ao horário de atendimento individual, disponibilizado pelo professor em um determinado dia da semana.

Percebe-se que, nesse curso analisado, poderia ter sido proposta, para além das atividades problematizadoras sugeridas pelo professor, a elaboração de um projeto de investigação estatística que possibilitasse aos alunos mobilizar os conhecimentos adquiridos para escolher um tema, definir um problema e investigá-lo. Cabe destacar que seria difícil incluir essa proposta na ementa, devido à carga horária do curso, que oferecia encontros semanais de três horas durante um semestre.

A formação inicial dos futuros professores de Matemática para atuar na Educação Básica precisa levar em consideração a necessidade de uma formação estatística que os habilite a elaborar atividades que promovam a aprendizagem estatística para além do uso de técnicas, superando os problemas apontados nas conclusões da pesquisa realizada por Costa (2007); ou seja, uma formação estatística que lhes permita inserir a estocástica em suas aulas e não os mantenha atrelados a livros didáticos e paradidáticos.

Nesse sentido, também é necessário investir na elaboração de materiais didáticos que subsidiem a formação inicial e contínua do professor, no que se refere à estatística.

A valorização do ensino de estatística e o diálogo com a matemática parecem ainda não ocorrer na nossa realidade escolar e nos cursos de formação de professores, pois os formandos chegam ao final da licenciatura, na grande maioria das vezes, sem condições para trabalhar a estatística em sala de aula (COSTA; NACARATO, 2011). 
Para alterar essa realidade educacional, é preciso buscar a elaboração de propostas centradas na concepção de estatística enquanto uma ciência de análise de dados e ter clareza sobre a interface dela com a matemática. A exploração da diversidade de situações que podem emergir dessa interseção, no curso de estatística, permitirá ao futuro professor mobilizar conceitos e procedimentos estatísticos para elaborar atividades de ensino para suas aulas na educação básica.

\section{Referências}

\section{ALIAGA, M.; et al. Guidelines for Assessment and Instruction in Statistics} Education (GAISE) - College report. Alexandria/VA/USA: American Statistical Association, 2010.

BAYER, A.; et al. Formandos em Matemática x Estatística na escola: estamos preparados? SIMPÓSIO SUL-BRASILEIRO DE ENSINO DE CIÊNCIAS, 12, 2004, Canoas. Atas... Canoas: ULBRA, 2004. v.1. p.1-12. Disponível em: <http:// www.exatas.net/ssbec_formandos_em_matematica.pdf>.Acesso em: 28 abr. 2012.

BRICKMAN, N. A.; TAYLOR, L. S. Aprendizagem activa. Lisboa: Fundação Calouste Gilbenkian. Serviço de Educação, 1996.

COBB, G. W.; MOORE, D. S. Mathematics, Statistics, and Teaching. The American Mathematical Monthly, Washington, v.104, n.9, p.801-823, nov.1997.

COSTA, A. A educação estatística na formação do professor de matemática. 2007. 153f. Dissertação (Mestrado em Educação) - Programa de Pós-Graduação Stricto Sensu em Educação, Universidade São Francisco, Itatiba, 2007.

COSTA, A.; NACARATO, A. A estocástica na formação do professor de matemática: percepções de professores e de formadores. Bolema, Rio Claro, v. 24, n. 39, p.367-386, nov. 2011. Disponível em: <http://www.periodicos.rc.biblioteca.unesp.br/index.php/ bolema/article/view/5092>. Acesso em: 30 abr. 2012.

COSTA, W. N. G.; PAMPLONA, A.S. Entrecruzando fronteiras: a Educação Estatística na formação de professores de Matemática. Bolema, Rio Claro, v. 24, n. 40, p. 897-911. 2011. Edição Temática: Educação Estatística.

FRANKLIN, C. Developing Graduate Programs in Statistics Education at the University of Georgia. ANNUAL MEETING OF THE AMERICAN STATISTICAL ASSOCIATION, $3^{\text {rd }}, 2002$, New York. Proceedings... Washington: American Statistical Association, 2002. p. 2972-2974. Disponível em: <http://www.amstat.org/sections/ srms/Proceedings/y2002/Files/JSM2002-000901.pdf>. Acesso em: 30 abr. 2012. 
FRANKLIN, C. et al. Guidelines for Assessment and Instruction in Statistics Education (GAISE) Report: a Pre-K-12 Curriculum Framework. Alexandria/VA: ASA, 2007.

LOPES, C. E. O ensino da estatística e da probabilidade na educação básica e a formação dos professores. Cad. Cedes, Campinas, v.28, n.74, p.57-73, jan./abr. 2008. Disponível em: <http://www.cedes.unicamp.br>. Acesso em: 30 abr. 2012.

LOPES, C.E. A probabilidade e a estatística no Ensino Fundamental: uma análise curricular. 1998. 133f. Dissertação (Mestrado em Educação) - Faculdade de Educação, Universidade de Campinas, Campinas, 1998.

Submetido em Novembro de 2012. Aprovado em Janeiro de 2013. 


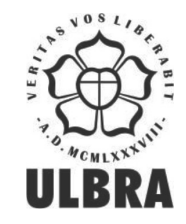

\section{ACTA SCIENTIAE}

\section{Revista de Ensino de Ciências e Matemática}

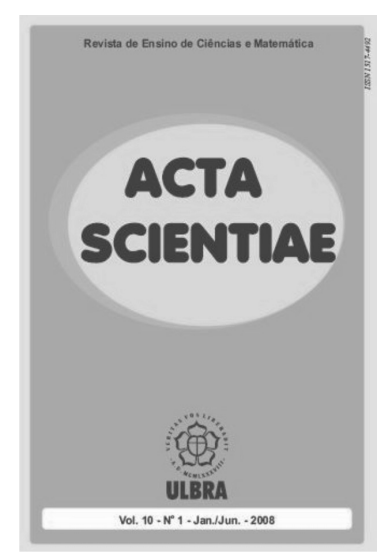

A Revista Acta Scientiae teve sua origem em 1999, mediante publicação de artigos oriundos dos pesquisadores das áreas de Ciências Naturais e Exatas da Universidade Luterana do Brasil - ULBRA Canoas (RS). Com sua indexação junto ao IBICT - Instituto Brasileiro de Informação em Ciência e Tecnologia (ISSN no. 1517-4492), é reconhecida como um espaço de publicação tanto de Ciências e Matemática como de Ensino dessas áreas. Entretanto, a partir do Volume 7, Número 1, 2005, Jan-Jun, a revista passa a publicar artigos exclusivos da área de Ensino de Ciências e Matemática, sendo editada desde sua fundação em dois números anuais. Assim, constitui-se em mais uma opção para publicação de artigos científicos dessa região de inquérito.

Confira: http://www.ulbra.br/actascientiae

Você poderá realizar download dos exemplares da revista, encontrará informações para submissão e avaliação dos artigos.

\section{Atenção!}

A Revista Acta Scientiae é de fluxo contínuo para o recebimento de artigos. Além disso, ela é uma revista de divulgação impressa e online.

\section{Informações:}

mauriciomatematica@gmail.com

actascientiae@ulbra.br 